\title{
Alu hypermethylation and high oxidative stress in patients with musculoskeletal tumors
}

\author{
Thamonwan Woraruthai $^{1}$, Chris Charoenlap $^{2}$, Chindanai Hongsaprabhas ${ }^{2}$, Apiwat Mutirangura ${ }^{3}$, Sittisak \\ Honsawek ${ }^{\text {Corresp. } 1}$ \\ ${ }^{1}$ Osteoarthritis and Musculoskeleton Research Unit, Department of Biochemistry, Faculty of Medicine, King Chulalongkorn Memorial Hospital, Thai Red \\ Cross Society, Chulalongkorn University, Bangkok, Thailand \\ 2 Department of Orthopaedics, Vinai Parkpian Orthopaedic Research Center, Faculty of Medicine, King Chulalongkorn Memorial Hospital, Thai Red Cross \\ Society, Chulalongkorn University, Bangkok, Thailand \\ 3 Center for Excellence in Molecular Genetics of Cancer \& Human Diseases, Department of Anatomy, Faculty of Medicine, King Chulalongkorn Memorial \\ Hospital, Thai Red Cross Society, Chulalongkorn University, Bangkok, Thailand \\ Corresponding Author: Sittisak Honsawek \\ Email address: sittisak.h@chula.ac.th
}

Background. Alu is one of the non-autonomous element retrotransposons, constituting nearly $11 \%$ of the human DNA. Methylation changes of the Alu element can cause genomic instability, a hallmark of cancer development, ultimately leading to the development of cancer. Epigenetic factors may induce the aberrant methylation of Alu and also oxidative stress. However, current knowledge of Alu methylation and oxidative stress is limited. There are few studies that have evaluated Alu methylation and oxidative stress on musculoskeletal tumor progression. Therefore, the present study evaluated the status of Alu methylation in musculoskeletal (MS) tumor, adjacent tissues, and blood leukocytes from MS tumor subjects, as well as unaffected participants. Moreover, we also investigated the oxidative stress status in MS tumor subjects and the control participants and determined the correlation between Alu methylation in MS tumors and that in blood leukocytes. Methods. Musculoskeletal tumors from musculoskeletal tumor patients $(n=$ $40)$ were compared to adjacent tissues $(n=40)$. The blood leukocytes from musculoskeletal tumor patients were compared to the blood leukocytes from controls ( $n=$ 107). Alu methylation status was analyzed using quantitative combined bisulfite restriction analysis (COBRA). In addition, 8-hydroxy 2'-deoxyguanosine (8-OHdG) values were determined using enzyme-linked immunosorbent assay. Results. Alu methylation values in MS tumors were statistically significantly higher than those in adjacent tissues $(P=$ 0.035). Similarly, Alu methylation statuses in the blood leukocytes of MS tumor subjects were statistically greater than those of control participants $(P<0.001)$. Moreover, there was a positive association between Alu methylation levels in MS tumors and blood leukocytes $(r=0.765, P<0.001)$. In addition, the highest tertile was significantly associated with the risk of $\mathrm{MS}$ tumors $(\mathrm{OR}=14.17,95 \% \mathrm{Cl}=5.08-39.51 ; P<0.001)$. The $8-\mathrm{OHdG}$ values in MS 
tumors were statistically higher than in adjacent tissues $(P<0.001)$ and circulating 8-OHdG levels were substantially greater in MS tumor subjects than in the control participants $(P<0.001)$. Discussion. These findings suggest that Alu methylation in blood leukocytes and plasma 8-OHdG might represent non-invasive biomarkers to help diagnose MS tumors. Therefore, Alu hypermethylation and high oxidative stress might be involved in the pathogenesis of the musculoskeletal tumors. 


\section{Manuscript Title:}

\section{Alu hypermethylation and high oxidative stress in patients with musculoskeletal tumors}

\section{Authors:}

4 Thamonwan Woraruthai ${ }^{1}$, Chris Charoenlap ${ }^{2}$, Chindanai Hongsaprabhas ${ }^{2}$, Apiwat Mutirangura ${ }^{3}$,

5 Sittisak Honsawek ${ }^{1, *}$

\section{Affiliations:}

$7{ }^{1}$ Osteoarthritis and Musculoskeleton Research Unit, Department of Biochemistry, Faculty of

8 Medicine, King Chulalongkorn Memorial Hospital, Thai Red Cross Society, Chulalongkorn

9 University, Bangkok, Thailand

10 2Department of Orthopaedics, Vinai Parkpian Orthopaedic Research Center, Faculty of

11 Medicine, King Chulalongkorn Memorial Hospital, Thai Red Cross Society, Chulalongkorn

12 University, Bangkok, Thailand

$13{ }^{3}$ Center for Excellence in Molecular Genetics of Cancer \& Human Diseases, Department of

14 Anatomy, Faculty of Medicine, King Chulalongkorn Memorial Hospital, Thai Red Cross

15 Society, Chulalongkorn University, Bangkok, Thailand.

\section{Corresponding Author:}

18 Sittisak Honsawek

19 Email: sittisak.h@chula.ac.th 


\section{Abstract}

Background. Alu is one of the non-autonomous element retrotransposons, constituting nearly $11 \%$ of the human DNA. Methylation changes of the Alu element can cause genomic instability,

23 a hallmark of cancer development, ultimately leading to the development of cancer. Epigenetic factors may induce the aberrant methylation of Alu and also oxidative stress. However, current knowledge of Alu methylation and oxidative stress is limited. There are few studies that have evaluated Alu methylation and oxidative stress on musculoskeletal tumor progression. Therefore, the present study evaluated the status of Alu methylation in musculoskeletal (MS) tumor, adjacent tissues, and blood leukocytes from MS tumor subjects, as well as unaffected participants. Moreover, we also investigated the oxidative stress status in MS tumor subjects and the control participants and determined the correlation between Alu methylation in MS tumors and that in blood leukocytes.

Methods. Musculoskeletal tumors from musculoskeletal tumor patients $(n=40)$ were compared to adjacent tissues $(n=40)$. The blood leukocytes from musculoskeletal tumor patients were compared to the blood leukocytes from controls $(n=107)$. Alu methylation status was analyzed using quantitative combined bisulfite restriction analysis (COBRA). In addition, 8-hydroxy 2'deoxyguanosine (8-OHdG) values were determined using enzyme-linked immunosorbent assay. Results. Alu methylation values in MS tumors were statistically significantly higher than those in adjacent tissues $(P=0.035)$. Similarly, Alu methylation statuses in the blood leukocytes of MS tumor subjects were statistically greater than those of control participants $(P<0.001)$. Moreover, 
41 leukocytes $(r=0.765, P<0.001)$. In addition, the highest tertile was significantly associated with

42 the risk of $\mathrm{MS}$ tumors $(\mathrm{OR}=14.17,95 \% \mathrm{CI}=5.08-39.51 ; P<0.001)$. The $8-\mathrm{OHdG}$ values in $\mathrm{MS}$ tumors

43 were statistically higher than in adjacent tissues $(P<0.001)$ and circulating 8-OHdG levels were

44 substantially greater in MS tumor subjects than in the control participants $(P<0.001)$.

45 Discussion. These findings suggest that Alu methylation in blood leukocytes and plasma 8-

$46 \mathrm{OHdG}$ might represent non-invasive biomarkers to help diagnose MS tumors. Therefore, Alu

47 hypermethylation and high oxidative stress might be involved in the pathogenesis of the

48 musculoskeletal tumors. 
Introduction

Musculoskeletal (MS) tumors are uncommon and distinct, as compared to other tumors.

Osteogenic and chondrogenic sarcomas account for approximately $0.5 \%$ of all malignancies in humans. Osteosarcoma affects mainly children and adolescents but most chondrosarcoma occurs predominantly in adults. The incidence of soft tissue sarcomas is 3 to 4 fold greater, and the majority of these patients are observed mainly after the 5th decade of life (Ofluoglu et al., 2010). The etiologies of MS tumors remain far from clear. Genetic and epigenetic factors could be involved in the pathogenesis of many tumors (Chalitchagorn et al., 2004). Moreover, a recent study suggests that epigenetic changes can be influenced by various factors such as age, environment, lifestyle, and disease states that can be related to tumor progression (Pogribny \& Beland, 2009).

There are 3 main processes of epigenetic change. One of these processes is DNA methylation; the cytosine base is modified at the $\mathrm{C} 5$ position $(5 \mathrm{mC})$ which inhibits the transcriptional process. However, this mechanism regulates the gene expression in the human genome, inactivates the $\mathrm{X}$ chromosome, affects the embryonic development, and most notably, suppresses the retrotransposon activity (Jin, Li \& Robertson, 2011). A methylation site in humans establishes retrotransposon elements including short interspersed nuclear elements or Alu, long interspersed nuclear elements-1 (LINE-1), and SAT-1. Alu is a non-autonomous retrotransposon, constituting nearly $11 \%$ of human DNA (Deininger et al., 2003). It can promote imperfect chromosome recombination, or insertions or deletions of the chromosomes (Mighell, Markham \& Robinson, 1997). Moreover, Alu can be inhibited by any DNA methylation process 
83 throughout its nucleotide sequences. A growing body of evidence suggests that methylation

84 changes in Alu can cause genomic instability, a hallmark of cancer development, ultimately

85 leading to the progression of cancer (Bae et al., 2012; Saito et al., 2010). Previous studies have

86 shown that Alu methylation of various cancers is lower than in control groups (Weisenberger et

87 al. 2005; Rhee et al., 2015; Jordà et al., 2017). However, a study reported that the percentage of

88 Alu methylated samples compared to a reference (PMR) in white blood cells from pancreatic

89 cancer patients was higher than in healthy controls (Neale et al., 2014). A previous study

revealed that methylation of retrotransposons was involved in the oxidative stress process which may be associated with musculoskeletal tumor progression (Donkena et al. 2010).

Oxidative stress is an imbalance of radicals and antioxidants that generate reactive oxygen species (ROS) in metabolic processes (Barzilai, Rotman \& Shiloh, 2002). ROS can cause DNA damage, including mutations, base modification, and DNA strand breakages (Valko et al., 2004). However, ROS cannot be disposed of by the body resulting in a diverse range of diseases, especially cancer (Halliwell \& Cross, 1994). One form of DNA damage is the 8-hydroxy 2'deoxyguanosine (8-OHdG), which is activated by oxidative DNA damage leading to base modification. 8-OHdG adducts can result in G-to-T transversions and mutations and the presence of 8-OHdG adducts in $\mathrm{CpG}$ islands strongly suppresses methylation of the adjacent cytosine (Franco et al., 2008). Thus, 8-OHdG can contribute to the aberrant DNA methylation process, leading to alteration of gene expression, genomic instability, and subsequently cancer progression (Ziech et al., 2011). Hence, 8-OHdG has widely served as a biomarker for oxidative stress. Current knowledge suggests that both oxidative stress and DNA methylation are factors that lead to diverse groups of cancer. Moreover, 8-OHdG levels can inhibit the DNA methylation process at the cytosine base, causing DNA hypomethylation. However, in the promoter region, 
106 8-OHdG can act as a catalyst for DNA methylation, leading to DNA hypermethylation (Franco 107 et al., 2008). In hepatocellular carcinoma, higher 8-OHdG levels were related to

108 hypermethylation of tumor suppressor genes (Nishida et al., 2013). Based on these observations, 109 Alu methylation in the human genome may be induced by increased oxidative DNA damage. There is limited information on the association between Alu methylation and oxidative

111 stress in musculoskeletal tumors progression. Therefore, the present study evaluated the Alu 112 methylation status in MS tumors, adjacent tissues, and blood leukocytes from MS tumor subjects 113 and unaffected volunteers. Moreover, we also investigated the oxidative stress status in MS 114 tumor subjects compared with the control participants and determined the correlation between 115 Alu methylation in musculoskeletal tumors and that in blood leukocytes.

117 Materials \& Methods

\section{Study population}

119 The experimental protocols were affirmed by the Ethical Committee on Human Research (IRB No. 439/59) of our institution. Written inform consent was provided by all participants in this 121 study.

This cross-sectional study had $40 \mathrm{MS}$ tumor patients between the ages 18-80 years who

123 had surgical treatment at the Department of Orthopedics, Chulalongkorn Memorial Hospital. MS

124 tumor specimens were collected from 11 patients with liposarcoma, 5 patients with

125 leiomyosarcoma, 4 patients with giant cell tumor, 4 patients with osteosarcoma, 4 patients with 126 chondrosarcoma, 4 patients with spindle cell tumor, 4 patients with lipoma, and 4 patients with 127 schwanoma. None of them had received preoperative systemic chemotherapy treatment. Tissues 128 from MS tumors and non-neoplastic adjacent tissues and venous blood samples were collected at 
129 the time of surgery. Histologically-normal, non-neoplastic adjacent tissues were at least $2 \mathrm{~cm}$

130 from the tumor margin. Venous blood samples were also collected from 107 healthy controls

131 between the ages 50-65 years who came for their annual health check-up at the hospital. Tissues,

132 including non-neoplastic adjacent tissues and neoplastic tissues, plasma, and blood leukocytes

133 were collected from all participants and stored at $-80^{\circ} \mathrm{C}$.

134 DNA extraction and quantitative combined bisulfite restriction analysis (COBRA)

135 Genomic DNA was isolated from tissues and peripheral blood mononuclear cells using a GF-1

136 nucleic acids extraction kit (Vivantis, Buckinghamshire, Malaysia). The concentration of DNA

137 samples was measured using a Nanodrop 2000 spectrophotometer (Thermo Scientific,

138 Wilmington, DE, USA). Then, $50 \mathrm{ng}$ of the extracted DNA was treated with bisulfite treatment

139 using an EZ DNA Methylation- Gold ${ }^{\mathrm{TM}}$ Kit (Zymo Research Corporation, Orange, CA, United

140 States), according to the manufacturer's protocol.

141 Alu methylation levels and patterns were analyzed using a COBRA Alu protocol

142 (Udomsinprasert et al., 2016). COBRA is a standard assay for determining Alu methylation

143 status (Jintaridth \& Mutirangura, 2010). The Alu sequence primers were based on the nucleotide

144 sequences in the regulatory region of the Alu sequence (the Alu Sx subfamily) (Batzer \&

145 Deininger, 2002). Briefly, bisulfited DNA samples were amplified with two specific primers for

146 COBRA Alu (Tiwawech et al., 2014), 5-GGRGRGGTGGTTTARGTTTGTAA-3 and 5-

147 CTAACTTTTTATATTTTTAATAAAAACRAAATTTCACCA-3. Each sample was quantitated

148 using the polymerase chain reaction (PCR), containing 10X PCR buffer (Qiagen, Germany), 200

$149 \mathrm{mM}$ dNTPs (Applied biosystem, United States), 25 mM MgCl2 (Qiagen, Germany), $20 \mu \mathrm{M}$

150 primers (forward and reverse primers), 0.5 U HotStar Taq DNA polymerase (Qiagen, Germany),

151 and $50 \mathrm{ng}$ bisulfited DNA. The PCR program was as follows: initial activation at $95^{\circ} \mathrm{C}$, followed 
152 by 40 cycles of $95^{\circ} \mathrm{C}$ for 45 seconds (denaturation process), $57^{\circ} \mathrm{C}$ for 45 seconds (annealing

153 process) and $72^{\circ} \mathrm{C}$ for 45 seconds (extension process), and the last step was at $72^{\circ} \mathrm{C}$ for 7

154 minutes (final extension process). After the amplification process, the PCR product was digested

155 with 2U Taq I restriction enzyme (Thermo scientific, United States) in Taq I buffer (Thermo

156 scientific, United States) and incubated at $65^{\circ} \mathrm{C}$ overnight. The cut PCR product was separated

157 on $8 \%$ nondenaturing polyacrylamide gels and followed by ethidium bromide staining. The

158 intensities of the DNA fragments were quatified using a Molecular Imager ${ }^{\circledR}$ Gel-Doc with Image

$159 \mathrm{Lab}^{\mathrm{TM}}$ Software (BioRad, Begonia Straat, Belgium).

160 Alu methylation analysis

161 The COBRA Alu was classified into 4 forms according to its methylation value of the two CpG

162 dinucleotides hereinafter: the hypermethylated form $\left({ }^{\mathrm{m}} \mathrm{C}^{\mathrm{m}} \mathrm{C}\right)$, the hypomethylated form $\left({ }^{\mathrm{u}} \mathrm{C}^{\mathrm{u}} \mathrm{C}\right)$,

163 and two forms of partial methylation $\left({ }^{\mathrm{m}} \mathrm{C}^{\mathrm{u}} \mathrm{C}\right.$ and $\left.{ }^{\mathrm{u}} \mathrm{C}^{\mathrm{m}} \mathrm{C}\right)$. Alu methylation levels and patterns were

164 analyzed to examine the actual percentage of methylated $\mathrm{CpG}$ dinucleotides. To calculate Alu

165 methylation status, the percentage of Alu methylation levels and patterns were analyzed in each

166 group according to the intensity of the COBRA-digested Alu products. The amplicons obtained

167 after enzymatic digestion of COBRA-Alu products were classified into 6 bands $(133,90,75,58$,

16843 , and $32 \mathrm{bp}$ ) which indicated distinct methylation statuses. The percentage of DNA

169 methylation within Alu was computed as the following: A = intensity of the 133-bp band divided

170 by $133 ; \mathrm{B}=$ intensity of the 58 -bp band divided by $58 ; \mathrm{C}=$ intensity of the $75-\mathrm{bp}$ band divided by

$17175 ; \mathrm{D}=$ intensity of the 90-bp band divided by $90 ; \mathrm{E}=$ intensity of the 43-bp band divided by 43 ;

172 and, $F=$ intensity of the 32-bp band divided by 32. The Alu methylation patterns were then

173 determined as the following: percentage of overall methylation loci

$174\left(\%{ }^{m} \mathrm{C}\right)=100 \times(\mathrm{E}+\mathrm{B}) /(2 \mathrm{~A}+\mathrm{E}+\mathrm{B}+\mathrm{C}+\mathrm{D}) ; \%{ }^{\mathrm{m}} \mathrm{C}^{\mathrm{m}} \mathrm{C}=100 \times \mathrm{F} /(\mathrm{A}+\mathrm{C}+\mathrm{D}+\mathrm{F}) ;$ 
17

176

177

178

179

180

181

182

183

184

185

186

187

188

189

190

191

192

193

194

195

196

197

$\%^{\mathrm{u}} \mathrm{C}^{\mathrm{m}} \mathrm{C}=100 \times \mathrm{C} /(\mathrm{A}+\mathrm{C}+\mathrm{D}+\mathrm{F}) ;\left(\%^{\mathrm{m}} \mathrm{C}^{\mathrm{u}} \mathrm{C}\right)=100 \times \mathrm{D} /(\mathrm{A}+\mathrm{C}+\mathrm{D}+\mathrm{F}) ;$ and

${ }^{\mathrm{u}} \mathrm{C}^{\mathrm{u}} \mathrm{C}=100 \times \mathrm{A} /(\mathrm{A}+\mathrm{C}+\mathrm{D}+\mathrm{F})$.

\section{8-hydroxy 2'-deoxyguanosine (8-OHdG) enzyme-linked immunosorbent assay (ELISA)}

Total DNA was extracted directly from tissues that were homogenized with liquid nitrogen and added to $10 \mu \mathrm{L}$ proteinase $\mathrm{K}$ and $400 \mu \mathrm{L}$ lysis buffer containing $50 \mathrm{mM}$ tris-hydrochloride (pH 7.4), $1 \mathrm{mM}$ ethylenediaminetetraacetic acid (EDTA) (pH 8.0), 0.5\% w/v sodium dodecylsulfate, and incubated at $50^{\circ} \mathrm{C}$ for 2 hours. After incubation, the lysates of the tissues were added to 250 $\mu \mathrm{L}$ phenol and $250 \mu \mathrm{L}$ chloroform:indole-3-acetic acid $\left(\mathrm{CHCl}_{3}: \mathrm{IAA} ; 49: 1\right)$. The lysates were centrifuged at $13,500 \mathrm{rpm}$ for 30 minutes at $4^{\circ} \mathrm{C}$. The supernatants were carefully transferred into a tube containing $4 \mu \mathrm{L}$ glycogen, $40 \mu \mathrm{L}$ sodium acetate, $800 \mu \mathrm{L}$ absolute ethanol and kept overnight at $-20^{\circ} \mathrm{C}$. The lysates were then centrifuged at $13,500 \mathrm{rpm}$ for 30 minutes at $4^{\circ} \mathrm{C}$. The supernatants were washed with $1 \mathrm{~mL} \mathrm{70 \%} \mathrm{ethanol} \mathrm{and} \mathrm{centrifuged} \mathrm{at} \mathrm{13,500} \mathrm{rpm} \mathrm{for} 5$ minutes at $4^{\circ} \mathrm{C}$. The supernatants were transferred into microcentrifuge tubes and put into a vacuum machine for 15 minutes before being dissolved with distilled water. The total DNA concentration was determined using a NanoDrop ${ }^{\circledR}$ ND-100 Spectrophotometer (Scientific, USA) and adjusted to $200 \mu \mathrm{g} / \mathrm{mL}$ DNA in each sample. Fasting venous blood samples from participants were collected in EDTA tubes to facilitate the isolation of plasma and leukocytes, and were then stored at $-80{ }^{\circ} \mathrm{C}$, if not measured immediately. $8-\mathrm{OHdG}$ values could be assessed using total DNA from tissue lysates and plasma of the participants and healthy controls by a commercially available HT 8-oxo-dG ELISA Kit II (Trevigen, Gaithersburg, United States), based on the instructions of the manufacturer. Antibodies specific to 8-OHdG generated by the entire immunogen were used. Twofold serial dilutions of 8-OHdG standard with a concentration of 0.89-56.7 ng/mL were applied as standards. Subsequently, the absorbance of the samples was 
198 measured at $450 \mathrm{~nm}$ using microplate reader. A standard optical density-concentration curve was

199 constructed for determination of 8-OHdG concentration in samples. Intra-assay and inter-assay

200 precisions were less than $10 \%$ and $15 \%$, respectively. The sensitivity of this assay was

$2010.57 \mathrm{ng} / \mathrm{mL}$.

\section{Statistical analysis}

203 Alu methylation levels between neoplastic and non-neoplastic adjacent tissues were determined

204 by the Wilcoxon signed-rank test. Alu methylation in blood leukocytes and healthy control

205 subjects was analyzed by the Mann-Whitney $U$-test. Moreover, the relationship between Alu

206 methylation status in neoplastic tissues and blood leukocytes was analyzed by Spearman's rank

207 correlation coefficient test. Linear regression analysis was conducted to analyze the potential of

208 Alu methylation levels as a biomarker. In addition, the relationship of Alu methylation levels and

209 musculoskeletal tumor risk was determined using logistic regression adjusted for age, gender,

210 and tumor size. The Mann-Whitney $U$-test was performed to determine the 8-OHdG values

211 between groups and Kruskal-Wallis H-test for continuous variables. A receiver operating

212 characteristic (ROC) curve was created to examine the area under the curve (AUC) for assessing

213 the practicability of applying Alu methylation level in blood leukocytes as a possible parameter

214 in distinguishing MS tumors subjects from the control participants. Statistical analysis was

215 performed using the Statistical Package for the Social Sciences (SPSS) version 20.0 (SPSS,

216 Inc.,Chicago, IL, United States) and figures were constructed using GraphPad Prism version 7.0.

217 Statistical significance was set at $P$ values $<0.05$.

218

219 Results

220 Hypermethylation of Alu in patients with musculoskeletal tumors 
221 The level of Alu methylation was assessed in 40 MS tumors, 40 adjacent tissues, and 107 healthy

222 controls. Alu methylation levels were examined in MS tumors compared to the adjacent tissues,

223 and Alu methylation in the blood leukocytes of MS tumor patients was compared to the control

224 subjects using COBRA Alu. The results revealed that the Alu element could be divided into 6

225 fragments: 133, 90, 75, 58, 43, and 32 bp (Figure 1).

226 When comparing Alu methylation levels in MS tumors with adjacent tissues, the results

227 showed that median Alu methylation status in MS tumors was statistically greater than that in the

228 adjacent tissues $(63.95 \%$ vs $58.84 \%, P=0.035)$ (Figure $2 A)$. Similarly, median Alu methylation

229 in blood leukocytes of MS tumor subjects was statistically greater than in the control participants

$230(71.23 \%$ vs $55.95 \%, P<0.001)$ (Figure $2 \mathrm{~B})$.

231 Association of Alu methylation levels in MS tumors and blood leukocytes

232 Alu methylation levels between MS tumors and blood leukocytes were analyzed to determine if

233 it could be used as a non-invasive biomarker. Interestingly, the results revealed that there was a

234 positive correlation between Alu methylation levels in MS tumors and blood leukocytes

$235(r=0.765, P<0.001)$ (Figure 3). Moreover, a ROC curve was evaluated to distinguish blood

236 leukocytes from patients with MS tumors from the controls. The area under the curve (AUC) for

237 Alu methylation in blood leukocytes was $0.832(95 \% \mathrm{CI}=0.746-0.918, P<0.001)$ (Figure 4). This

238 model has a threshold cut-off value of 60.274 , sensitivity of 0.825 and specificity of 0.759 .

239 Relationship of Alu methylation and susceptibility of MS tumors

240 To evaluate Alu methylation status in peripheral blood mononuclear cells and the susceptibility

241 to MS tumors, logistic regression was performed. As displayed in Table 1, the results indicated

242 that overall Alu methylation levels increased, reflecting a higher occurring risk for MS tumors

$243(\mathrm{OR}=1.13,95 \% \mathrm{CI}=1.08-1.18 ; P<0.001)$. Furthermore, Alu methylation distribution was 
244 categorized into low methylation and high methylation. Individuals with high Alu methylation

245 above the cut-off value $(>60.274)$ were associated with a 12.8 -fold $(95 \% \mathrm{CI}=5.46-30.02$;

$246 P<0.001)$ increased risk of MS tumors, compared to individuals with low Alu methylation. When

247 a dose-response effect was determined by tertile, the results showed that Alu hypermethylation

248 was associated with an increased risk for MS tumors. In addition, the highest tertile was

249 significantly associated with the risk for $\mathrm{MS}$ tumors $(\mathrm{OR}=14.17,95 \% \mathrm{CI}=5.08-39.51 ; P<0.001)$.

250 Elevated levels of 8-OHdG in tumor specimens and plasma from MS tumor patients

251 In this study, plasma 8-OHdG levels were evaluated in 107 controls and $40 \mathrm{MS}$ tumor patients.

252 8-OHdG levels were assessed in the tissues and plasma. The results revealed that the median 8-

253 OHdG value in MS tumor specimens was substantially elevated compared with the adjacent

254 tissues $(P<0.001)$ (Figure 5A). Similarly, the median plasma 8-OHdG level in MS tumor

255 subjects was also markedly greater than in the control participants $(P<0.001)$ (Figure 5B).

256 However, there was no correlation between Alu methylation levels and 8-OHdG levels in the

257 tissues and plasma of MS tumor patients.

258

259 Discussion

260 Research in recent years has shown that epigenetic changes lead to pathogenesis in many

261 cancers. In this study, we investigated the Alu methylation levels in MS tumors and adjacent

262 tissues and in blood leukocytes of MS tumor patients and healthy controls. In addition, this study

263 determined the 8-OHdG levels in MS tumors and adjacent tissues as well as plasma 8-OHdG

264 levels. This study showed that the Alu methylation levels in MS tumors were higher than in

265 adjacent tissues. Also, the Alu element was hypermethylated in blood leukocytes of MS tumor

266 patients. Moreover, there was a positive relationship between Alu methylation status in 
267 peripheral blood leukocytes and MS tumors. Notably, Alu methylation values in peripheral blood

268 leukocytes were associated with MS tumor risk. As for the 8-OHdG levels, the results showed

269 that 8-OHdG levels in MS tumors and plasma of patients with MS tumors were higher than in

270 the controls. Therefore, these results propose that epigenetic alteration may play a role in MS

271 tumor progression.

272 A recent study revealed that repetitive elements methylation can cause alteration of gene 273 expression, leading to genomic instability (Udomsinprasert et al., 2016). Currently, our study is

274 the first that has investigated Alu methylation levels in tumors and blood leukocytes of MS

275 tumor patients and also determined a correlation between blood leukocytes and MS tumors.

276 Moreover, both 8-OHdG levels in plasma and MS tumors were determined. Previous

277 investigations have reported that aberrant methylation is associated with several types of cancers,

278 including lung, breast, colon, and gastric cancers (Kim et al., 2014). Hypomethylation of

279 repetitive elements has been shown to be associated with an increased risk of several types of

280 cancers, whereas other investigations revealed that hypermethylation of these elements were

281 associated with cancer. For example, the Alu methylation levels in white blood cells of

282 colorectal cancer patients were higher than those in the controls (Rhiannon et al., 2013).

283 Likewise, the Alu methylation status in white blood cells of pancreatic cancer patients was also

284 higher than in the controls (Neale et al., 2014). These findings demonstrated that the elevated

285 percentage of methylated samples compared to a reference Alu methylation status in cancer

286 subjects was associated with pancreatic cancer risk (Neale et al., 2014). Using other assays to

287 determine the DNA methylation levels has discovered that the global methylation levels in

288 peripheral blood DNA are significantly higher in breast cancer patients than in the controls $(\mathrm{Xu}$

289 et al., 2012). In addition, LINE-1 methylation levels are significantly higher in renal cell 
290 carcinoma patients compared to the controls, and LINE-1 hypermethylation is positively related

291 with an elevated risk of renal cell cancer (Liao et al., 2011). Furthermore, endogenous double-

292 strand breaks in cancer cells contain higher DNA methylation levels than the cellular genome

293 (Pornthanakasem et al., 2008). The relationship between Alu methylation in blood leukocytes

294 and MS tumor risk may be associated with increased methylation around the DNA double-strand

295 break region. However, the majority of the previous investigations have reported that DNA

296 hypomethylation was related to the risk of several type of cancers such as ovarian cancer (Akers

297 et al., 2014), head and neck cancer (Langevin et al., 2012), and breast cancer (Wu et al., 2012).

298 These findings report that repetitive elements in patients with cancer are hypomethylated when

299 compared to the controls. Previous studies have also demonstrated that Alu methylation levels in

300 blood leukocytes of breast and pancreatic cancer patients are significantly lower than those of

301 controls (Wu et al., 2012; Dauksa et al., 2012). Additionally, aberrant DNA methylation (either

302 hypermethylation or hypomethylation) can lead to genomic instability and cancer progression

303 (Esteller M, 2007). The possible explanation for this discrepancy remains unclear and it may be

304 due to the differences in cancers, cell types, and/or methodologies used to assess the methylation

305 levels. Several methods to measure methylation level include real-time PCR-based methods,

306 sequencing-based methods, and gel electrophoresis-based quantitative methods (COBRA PCR).

307 Our protocol of COBRA PCR targets shorter amplicon sizes of the Alu sequences, which

308 greatly improve yield when amplifying genetic material derived from cells or tissues. We have

309 validated this method by comparing it to pyrosequencing and found that interspersed repetitive

310 sequence-COBRA was very accurate and reliable (Jintaridth \& Mutirangura, 2010).

311 Interestingly, the findings from this study demonstrated that Alu methylation levels of

312 MS tumors were positively correlated with blood leukocytes. We also found that the Alu 
313 methylation could be a promising biomarker because it yielded the proper differentiation power

314 between the MS tumors and the controls. By setting the cut-off value and applying it, we could

315 develop an indicator helpful for distinguishing the MS tumor patients from the controls. We do

316 not recommend that this test be used for confirmation of MS tumors because the

317 histopathological study should remain the gold standard for a definitive diagnosis. However, Alu

318 methylation in blood leukocytes might be utilized as a non-invasive blood-based biomarker for

319 monitoring the severity and progression of MS tumors.

320 DNA hypomethylation is believed to result in chromosomal instability by allowing

321 silenced areas of the genome, such as retrotransposons, to become active (Chen et al., 1998).

322 Conversely, our findings revealed that Alu hypermethylation in blood leukocytes was associated

323 with MS tumor risk. Whether it is Alu hypomethylation or hypermethylation that was associated

324 with cancer risk, the mechanism underlying this relationship between aberrant DNA methylation

325 and carcinogenesis remain uncertain. The association we have observed between Alu

326 hypermethylation and MS tumor risk might be related to a high frequency of double-strand

327 breaks/ oxidative DNA damage in individuals with tumor, however, future investigations will be

328 needed to validate this assumption.

We observed, 8-OHdG levels of the plasma from MS tumor patients were higher than in controls. In addition, 8-OHdG levels of tumor tissues were significantly higher than the adjacent tissues. This finding is consistent with a previous study that showed elevated oxidative stress was associated with the mortality of patients and that increased serum insulin-like growth factor-1 levels can prevent musculoskeletal tumors from occurring (Elis et al., 2011). Recent studies have

334 also reported that 8-OHdG values in cancer subjects are greater than in control participants

335 (Mohamadkhani et al., 2017; Ma-On et al., 2015). These results suggest that increased 8-OHdG 
336 levels might be a parameter of high oxidative stress, defective antioxidant protection and/or

337 deficient repair of oxidative DNA damage. Furthermore, 8-OHdG levels could suppress DNA

338 methylation at nearby cytosine bases leading to

339 DNA hypomethylation ( $\mathrm{Wu} \& \mathrm{Ni}, 2015)$. Moreover, 8-OHdG is potentially one of the most

340 abundant DNA lesions formed by oxidative stress and this mutagenic lesion results in base

341 transversions (Lunec et al., 2002). Therefore, 8-OHdG is involved in the progression of cancer

342 via 8-OHdG adduction. In the current study, there were no correlations between Alu methylation

343 levels and 8-OHdG levels in both plasma and tumor tissues. These findings suggest that

344 oxidative stress was not directly related to repetitive element methylation but oxidative stress

345 could somehow be associated with the pathogenesis of MS tumor.

346 This is a cross-sectional study, and therefore, it has some limitations. First, the MS tumor

347 types were heterogenous. Hence, it is challenging to analyze the Alu methylation levels data for

348 each subgroup. Second, the sample size in this study was relatively small, so the authors cannot

349 conclude with certainty that the Alu methylation levels in each subgroup are higher than in the

350 control group. Additional studies with a larger sample size are required. Third, DNA methylation

351 is an epigenetic mechanism so it is possible that confounding factors such as lifestyle, diet,

352 alcohol drinking, smoking, and body mass index (BMI) may have affected the Alu methylation

353 levels of MS tumor patients. Fourth, the authors could not analyze the association between Alu

354 methylation levels and the clinical outcomes because complete clinical data was not available in

355 our database. Due to the design of this study, there was no long-term follow-up of the MS tumor

356 patients' symptoms. It is recommended that a prospective study with a long follow-up period

357 could be conducted to further investigate Alu methylation levels and the risk of developing MS

358 tumors. Additionally, incomplete assessment of tumor subtype, tumor stage or grade needs to be 
359 taken into consideration due to limitations of record accessibility. Other caveats would be the

360 lack of serum C-reactive protein value. Future study should collect these data to further examine

361 the differences between subgroups.

\section{Conclusion}

363 To summarize, our study illustrated that Alu hypermethylation and elevated 8-OHdG status

364 were evident in MS tumor tissues and that Alu hypermethylation and high oxidative stress

365 were present even in peripheral blood of MS tumor patients. To our knowledge, this study is the

366 first to demonstrate the association between Alu methylation in the MS tumors and in the blood

367 leukocytes, indicating that Alu methylation could be considered as a possible non-invasive

368 blood-based marker when diagnosing MS tumors. We also suggest that Alu hypermethylation

369 might reflect the severity of an epigenetic field for tumorigenesis and could become an

370 epigenetic biomarker for the tumor risk prediction, monitoring, and follow-up of MS tumor

371 patients. Additional research is warranted using prospective cohort designs to affirm this finding,

372 further unravel a causal and/or correlative relationship and to yield more evidence for the utility

373 of examining Alu methylation in blood leukocytes as a potential biomarker of MS tumors.

\section{Acknowledgements}

376 The authors would like to thank the Department of Biochemistry and Chula Medical Research

377 Center (Chula MRC), Faculty of Medicine, Chulalongkorn University, for providing the

378 facilities. The authors acknowledge the staff of Center for Excellence in Molecular Genetics of

379 Cancer \& Human Diseases and the Thailand Research Fund (DPG5980005) for their technical

380 assistance. We thank June Ohata, Tom Mabey, and Ellie McConachie for reviewing and proof-

381 reading the manuscript. 


\section{References}

Akers SN, Moysich K, Zhang W, Collamat Lai G, Miller A, Lele S, Odunsi K, Karpf AR. 2014.

LINE1 and Alu repetitive element DNA methylation in tumors and white blood cells from epithelial ovarian cancer patients. Gynecologic Oncology, 132: 462-467. DOI 10.1016/j.ygyno.2013.12.024.

Bae JM, Shin SH, Kwon HJ, Park SY, Kook MC, Kim YW, Cho NY, Kim N, Kim TY, Kim D,

Kang GH. 2012. ALU and LINE-1 hypomethylations in multistep gastric carcinogenesis and their prognostic implications. International Journal of Cancer, 131: 1323-1331. DOI 10.1002/ijc.27369.

Barzilai A, Rotman G, Shiloh Y. 2002. ATM deficiency and oxidative stress: a new dimension of defective response to DNA damage. DNA Repair (Amst), 1: 3-25.

Batzer MA, Deininger PL. 2002. Alu repeats and human genomic diversity. Nat Rev Genet, 395 3:370-379. DOI 10.1038/nrg798.

Chalitchagorn K, Shuangshoti S, Hourpai N, Kongruttanachok N, Tangkijvanich P, Thong-ngam

D, Voravud N, Sriuranpong V, Mutirangura A. 2004. Distinctive pattern of LINE-1 methylation

level in normal tissues and the association with carcinogenesis. Oncogene, 23: 8841-8846.

399 Chen RZ, Pettersson U, Beard C, Jackson-Grusby L, Jaenisch R. 1998. DNA hypomethylation

400 leads to elevated mutation rates. Nature, 395: 89-93. DOI 10.1038/25779.

401 Dauksa A, Gulbinas A, Barauskas G, Pundzius J, Oldenburg J, El-Maarri O. 2012. Whole blood 402 DNA aberrant methylation in pancreatic adenocarcinoma shows association with the course of 403 the disease: a pilot study. PLoS One, 7: e37509. DOI 10.1371/journal.pone.0037509. 
404 Deininger PL, Moran JV, Batzer MA, Kazazian HH Jr. 2003. Mobile elements and mammalian 405 genome evolution. Current Opinion in Genetics \& Development, 13: 651-658.

406 Donkena KV, Young CY, Tindall DJ. 2010. Oxidative stress and DNA methylation in prostate 407 cancer. Obstetrics and Gynecology International, 2010: 302051 DOI 10.1155/2010/302051.

408 Elis S, Wu Y, Courtland HW, Sun H, Rosen CJ, Adamo ML, Yakar S. 2011. Increased serum 409 IGF-1 levels protect the musculoskeletal system but are associated with elevated oxidative stress 410 markers and increased mortality independent of tissue igfl gene expression. Aging Cell, 10: 547411 550. DOI 10.1111/j.1474-9726.2011.00683.x.

412 Esteller M. 2007. Cancer epigenomics: DNA methylomes and histone-modification maps. Nat 413 Rev Genet, 8: 286-298. DOI 10.1038/nrg2005.

414 Franco R, Schoneveld O, Georgakilas AG, Panayiotidis MI. 2008. Oxidative stress, DNA 415 methylation and carcinogenesis. Cancer Letters, 266: 6-11 DOI 10.1016/j.canlet.2008.02.026.

416 Halliwell B, Cross CE. 1994. Oxygen-derived species: their relation to human disease and 417 environmental stress. Environmental Health Perspectives, 102 Suppl 10:5-12.

418 Jin B, Li Y, Robertson KD. 2011. DNA methylation: superior or subordinate in the epigenetic 419 hierarchy?. Genes Cancer, 2: 607-617. DOI 10.1177/1947601910393957.

420 Jintaridth P, Mutirangura A. 2010. Distinctive patterns of age-dependent hypomethylation in 421 interspersed repetitive sequences. Physiol Genomics, 41: 194-200. DOI

422 10.1152/physiolgenomics.00146.2009.

423 Jordà M1, Díez-Villanueva A, Mallona I, Martín B, Lois S, Barrera V, Esteller M, Vavouri T, 424 Peinado MA. 2017. The epigenetic landscape of Alu repeats delineates the structural and 425 functional genomic architecture of colon cancer cells. Genome Research, 27: 118-132. DOI 426 10.1101/gr.207522.116. 
427 Kim B, Kang S, Jeong G, Park SB, Kim SJ. 2014. Identification and comparison of aberrant key

428 regulatory networks in breast, colon, liver, lung, and stomach cancers through methylome

429 database analysis. PLoS One, 9:e97818. DOI 10.1371/journal.pone.0097818.

430 Langevin SM, Koestler DC, Christensen BC, Butler RA, Wiencke JK, Nelson HH, Houseman

431 EA, Marsit CJ, Kelsey KT. 2012. Peripheral blood DNA methylation profiles are indicative of

432 head and neck squamous cell carcinoma: an epigenomewide association study. Epigenetics, 7:

433 291-299. DOI 10.4161/epi.7.3.19134.

434 Liao LM, Brennan P, van Bemmel DM, Zaridze D, Matveev V, Janout V, Kollarova H, Bencko

435 V, Navratilova M, Szeszenia-Dabrowska N, Mates D, Rothman N, Boffetta P, Chow WH, Moore

436 LE. 2011. LINE-1 methylation levels in leukocyte DNA and risk of renal cell cancer. PLoS ONE

437 6:e27361. DOI 10.1371/journal.pone.0027361.

438 Lunec J, Holloway KA, Cooke MS, Faux S, Griffiths HR, Evans MD. 2002. Urinary 8-oxo-2'-

439 deoxyguanosine: redox regulation of DNA repair in vivo?. Free Radical Biology \& Medicine, $440 \quad 33: 875-885$.

441 Ma-On C, Sanpavat A, Whongsiri P, Suwannasin S, Hirankarn N, Tangkijvanich P, Boonla C.

442 2017. Oxidative stress indicated by elevated expression of $\mathrm{Nrf2}$ and 8-OHdG promotes

443 hepatocellular carcinoma progression. Medical Oncology, 34: 57. DOI 10.1007/s12032-017-

444 0914-5.

445 Mighell AJ, Markham AF, Robinson PA. 1997. Alu sequences. FEBS Letters, 417: 1-5.

446 Mohamadkhani A, Pourshams A, Viti J, Cellai F, Mortazavi K, Sharafkhah M, Sotoudeh M,

447 Malekzadeh R, Boffetta P, Peluso M. 2017. Pancreatic Cancer is Associated with Peripheral

448 Leukocyte Oxidative DNA Damage. Asian Pacific Journal of Cancer Prevention, 18: 1349-1355. 
449 Neale RE, Clark PJ, Fawcett J, Fritschi L, Nagler BN, Risch HA, Walters RJ, Crawford WJ,

450 Webb PM, Whiteman DC, Buchanan DD. 2014. Association between hypermethylation of DNA

451 repetitive elements in white blood cell DNA and pancreatic cancer. Cancer Epidemiology, 38 :

452 576-582. DOI 10.1016/j.canep.2014.08.006.

453 Nishida N, Arizumi T, Takita M, Kitai S, Yada N, Hagiwara S, Inoue T, Minami Y, Ueshima K,

454 Sakurai T, Kudo M. 2013. Reactive oxygen species induce epigenetic instability through the

455 formation of 8-hydroxydeoxyguanosine in human hepatocarcinogenesis. Digestive Diseases, 31 :

456 459-466. DOI 10.1159/000355245.

457 Ofluoglu O, Boriani S, Gasbarrini A, De Iure F, Donthineni R. 2010. Diagnosis and planning in

458 the management of musculoskeletal tumors: surgical perspective. Seminars in Interventional

459 Radiology, 27:185-190. DOI 10.1055/s-0030-1253516.

460 Pogribny IP, Beland FA. 2009. DNA hypomethylation in the origin and pathogenesis of human

461 diseases. Cellular and Molecular Life Sciences, 66: 2249-2261. DOI 10.1007/s00018-009-00154625.

463 Pornthanakasem W, Kongruttanachok N, Phuangphairoj C, Suyarnsestakorn C, Sanghangthum

464 T, Oonsiri S, Ponyeam W, Thanasupawat T, Matangkasombut O, Mutirangura A. 2008. LINE-1

465 methylation status of endogenous DNA double-strand breaks. Nucleic Acids Res, 36:3667-3675.

466 DOI http://dx.doi.org/10.1093/nar/gkn261.

467 Rhee YY, Lee TH, Song YS, Wen X, Kim H, Jheon S, Lee CT, Kim J, Cho NY, Chung JH,

468 Kang GH. 2015. Prognostic significance of promoter CpG island hypermethylation and

469 repetitive DNA hypomethylation in stage I lung adenocarcinoma. Virchows Archiv. 466: 675-

470 683. DOI 10.1007/s00428-015-1749-0. 
471 Saito K, Kawakami K, Matsumoto I, Oda M, Watanabe G, Minamoto T. 2010. Long interspersed 472 nuclear element 1 hypomethylation is a marker of poor prognosis in stage IA non-small cell lung 473 cancer. Clinical Cancer Research, 16: 2418-2426. DOI 10.1158/1078-0432.CCR-09-2819.

474 Udomsinprasert W, Kitkumthorn N, Mutirangura A, Chongsrisawat V, Poovorawan Y, 475 Honsawek S. 2016. Global methylation, oxidative stress, and relative telomere length in biliary 476 atresia patients. Scientific Reports, 6: 26969 DOI 10.1038/srep26969.

477 Valko M, Izakovic M, Mazur M, Rhodes CJ, Telser J. 2004. Role of oxygen radicals in DNA 478 damage and cancer incidence. Molecular and Cellular Biochemistry, 266: 37-56.

479 Walters RJ, Williamson EJ, English DR, Young JP, Rosty C, Clendenning M, Walsh MD, Parry 480 S, Ahnen DJ, Baron JA, Win AK, Giles GG, Hopper JL, Jenkins MA, Buchanan DD. 2013. 481 Association between hypermethylation of DNA repetitive elements in white blood cell DNA and 482 early-onset colorectal cancer. Epigenetics, 8: 748-755 DOI 10.4161/epi.25178.

483 Weisenberger DJ, Campan M, Long TI, Kim M, Woods C, Fiala E, Ehrlich M, Laird PW. 2005. 484 Analysis of repetitive element DNA methylation by MethyLight. Nucleic Acids Research, 33 : $485 \quad 6823-6836$.

486 Wu HC, Delgado-Cruzata L, Flom JD, Perrin M, Liao Y, Ferris JS, Santella RM, Terry MB. 487 2012. Repetitive element DNA methylation levels in white blood cell DNA from sisters 488 discordant for breast cancer from the New York site of the Breast Cancer Family Registry. 489 Carcinogenesis 33: 1946-1952 DOI 10.1093/carcin/bgs201.

490 Wu Q, Ni X. 2015. ROS-mediated DNA methylation pattern alterations in carcinogenesis. 491 Current Drug Targets, 16: 13-9.

492 Xu X, Gammon MD, Hernandez-Vargas H, Herceg Z, Wetmur JG, Teitelbaum SL, Bradshaw 493 PT, Neugut AI, Santella RM, Chen J. 2012. DNA methylation in peripheral blood measured by 
494 LUMA is associated with breast cancer in a population-based study. The FASEB Journal, 26:

495 2657-2666 DOI 10.1096/fj.11-197251.

496 Ziech D, Franco R, Pappa A, Panayiotidis MI. 2011. Reactive oxygen species (ROS)--induced

497 genetic and epigenetic alterations in human carcinogenesis. Mutation Research, 711: 167-173

498 DOI 10.1016/j.mrfmmm.2011.02.015.

499

500

501 


\section{Figure 1}

\section{Alu methylation of participants}

$8 \%$ non-denaturing polyacrylamide gel of Alu elements in adjacent tissues, MS tumors and blood leukocytes, respectively $(M=25$ bp DNA marker, $A=$ adjacent tissues, $T=M S$ tumors and $\mathrm{B}=$ blood leukocytes).

*Note: Auto Gamma Correction was used for the image. This only affects the reviewing manuscript. See original source image if needed for review.

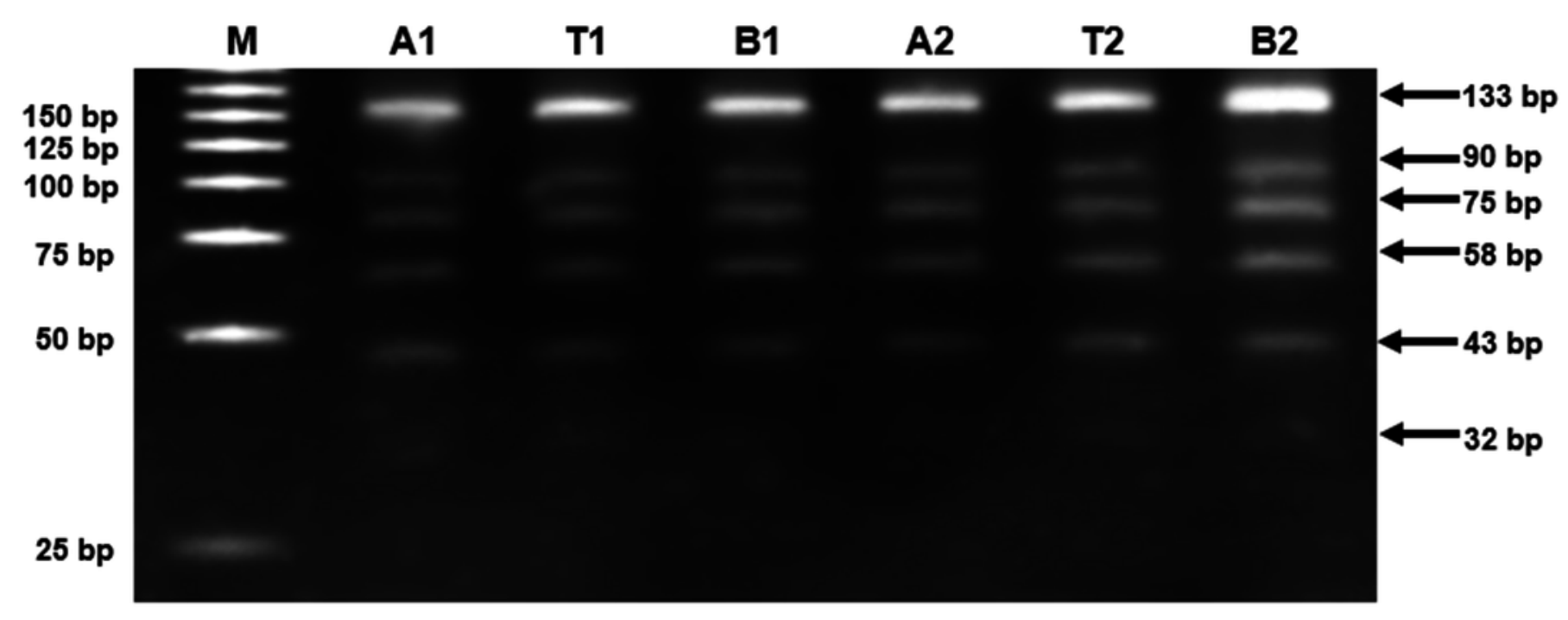


Figure 2

Percentage of Alu methylation levels in MS tumor patients and controls.

(A) Alu methylation levels in adjacent tissues and MS tumors, (B) Alu methylation levels in blood leukocytes of MS tumor patients compared with controls.

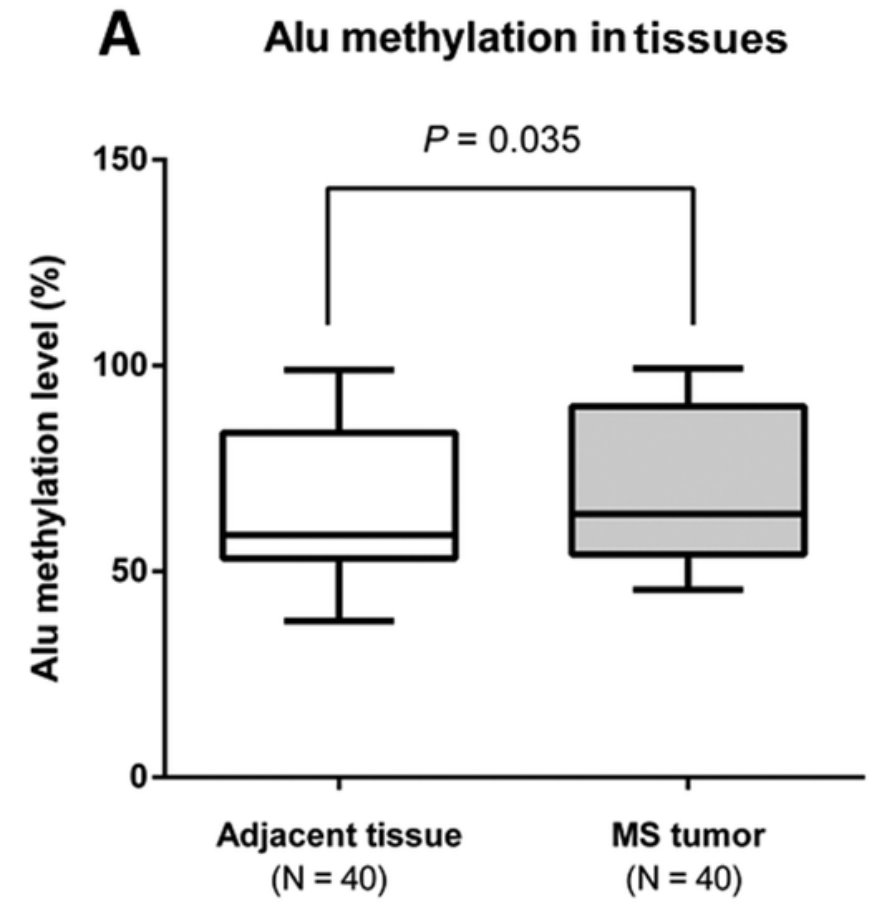

B Alu methylation in blood leukocytes

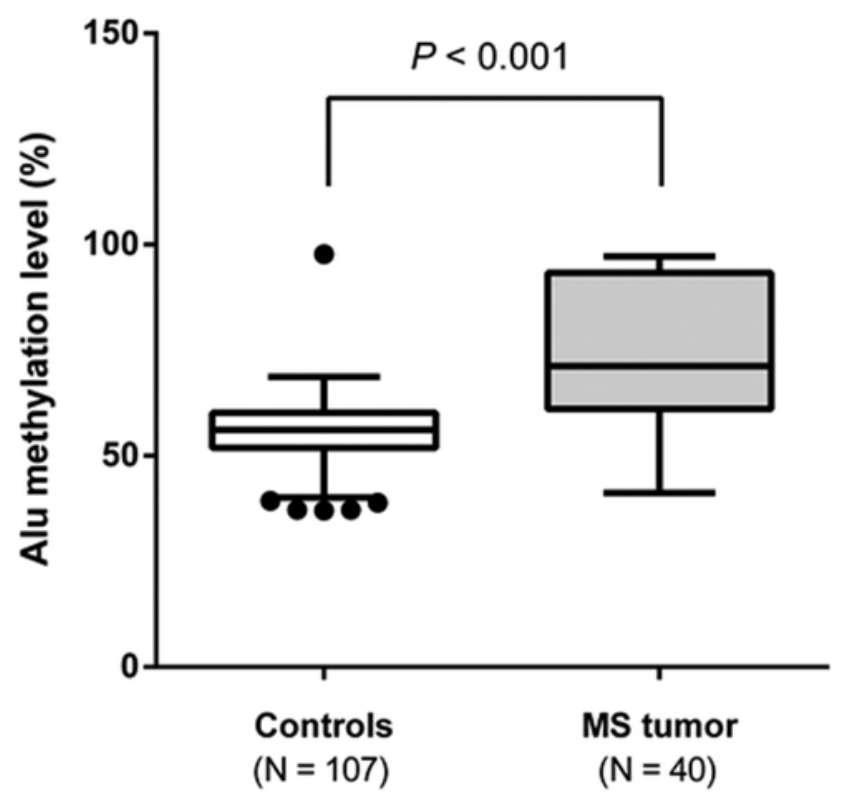


Figure 3

Positive correlation between Alu methylation levels in MS tumor and blodd leukocytes.

Each data point indicates the correlation between Alu methylation levels in MS tumor and blodd leukocytes.

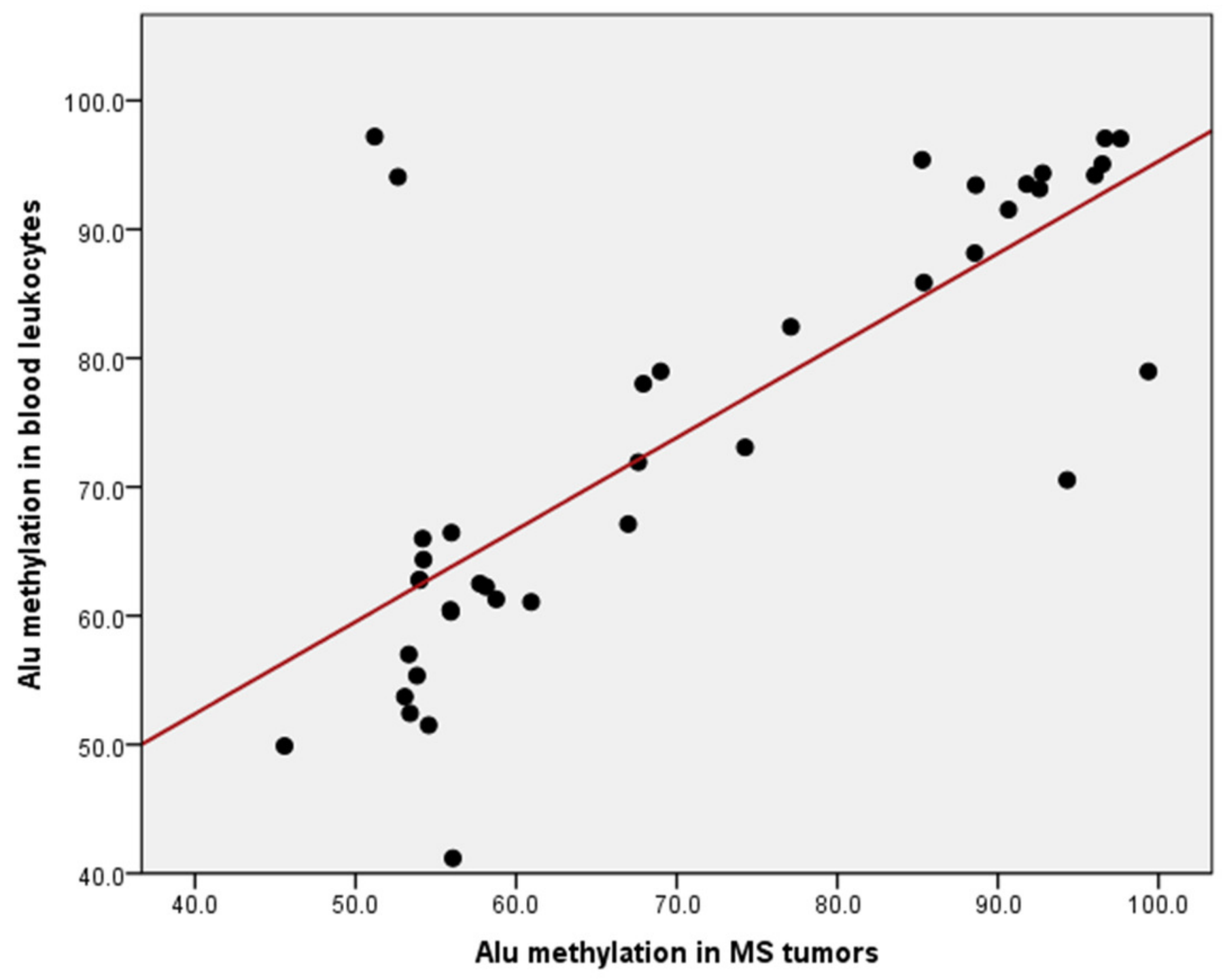


Figure 4

ROC curve analysis of Alu methylation levels in blood leukocytes that distinguish MS tumor from controls.

ROC curve analysis of Alu methylation levels in blood leukocytes that distinguish MS tumor from controls.

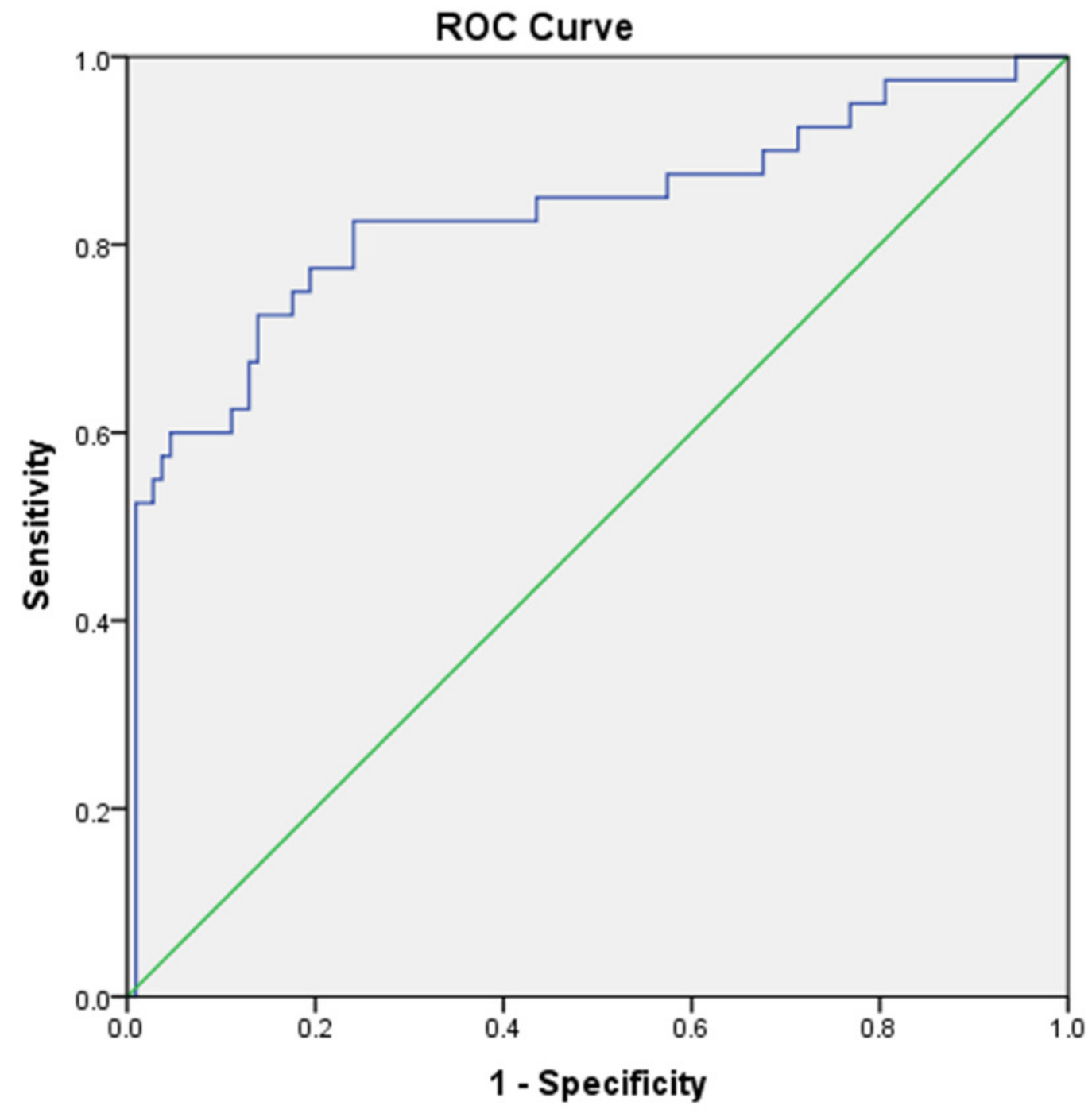


Figure 5

8-OHdG levels in MS tumor patients and controls.

(A) 8-OHdG levels in MS tumors and adjacent tissues, (B) Plasma 8-OHdG levels in MS tumor patients compared with controls.
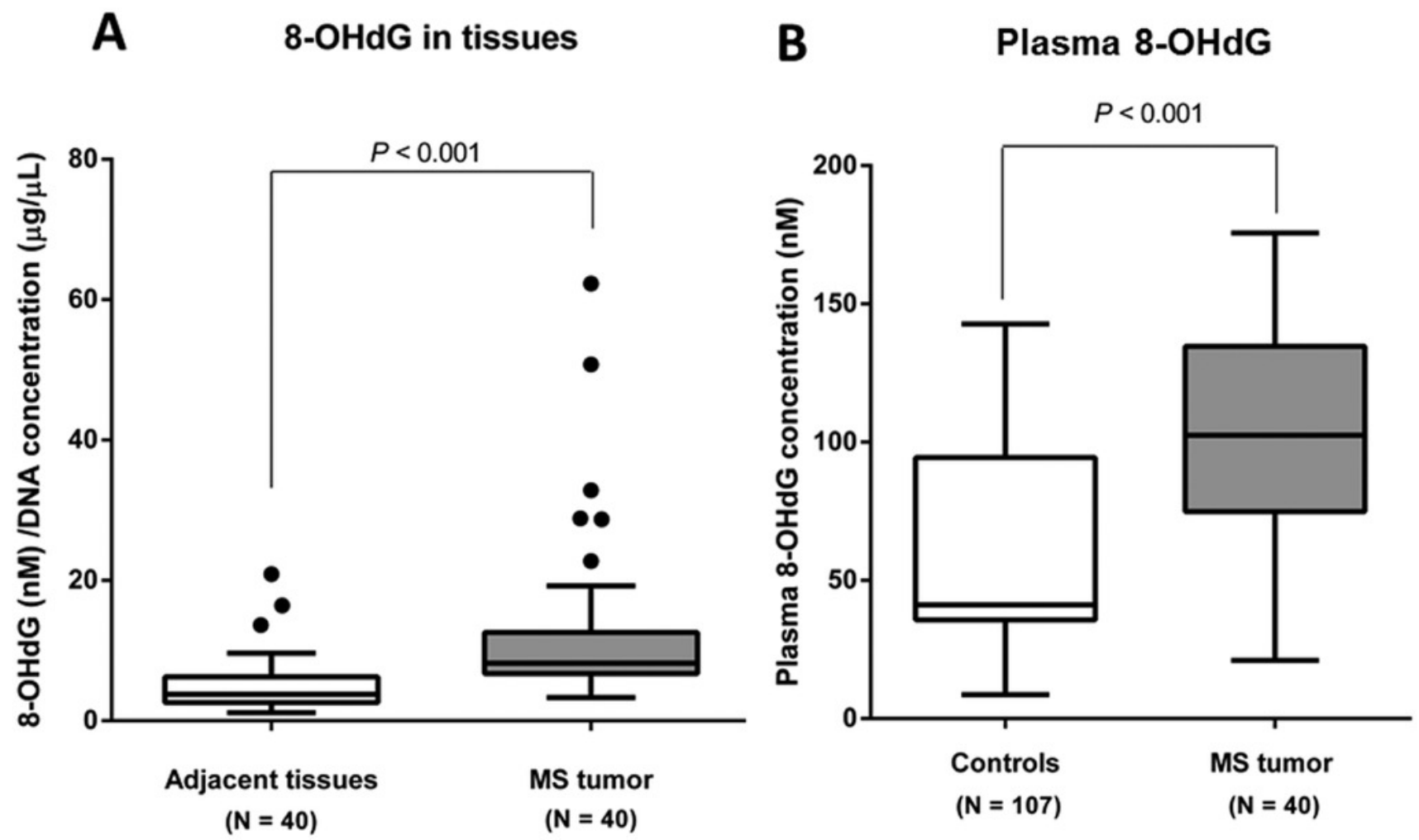


\section{Table $\mathbf{1}$ (on next page)}

Association between Alu methylation and risk of MS tumor.

Data was presented as median \pm SEM. $P$-value $<0.05$ indicates a significant difference of Alu methylation levels between MS tumor patients and controls. 
1

2

3

4

5

6

7

8

9

10

11

12

13

\begin{tabular}{|c|c|c|c|c|}
\hline Repetitive elements & $\begin{array}{l}\text { MS tumor } \\
(\mathbf{N}=\mathbf{4 0})\end{array}$ & $\begin{array}{l}\text { Controls } \\
(\mathbf{N}=107)\end{array}$ & OR $(95 \% \mathrm{CI})$ & $P$-value \\
\hline \multicolumn{5}{|l|}{ Alu element } \\
\hline Overall & $100 \%$ & $100 \%$ & $1.13(1.08-1.18)$ & $<0.001$ \\
\hline \multicolumn{5}{|l|}{ By cut-off value } \\
\hline Low methylation & $19.56 \%$ & $75.70 \%$ & 1.00 (reference) & \\
\hline High methylation & $80.44 \%$ & $24.30 \%$ & $12.80(5.46-30.02)$ & $<0.001$ \\
\hline \multicolumn{5}{|l|}{ By tertile } \\
\hline $1^{\text {st }}$ tertile & $27.10 \%$ & $33.33 \%$ & 1.00 (reference) & \\
\hline $2^{\text {nd }}$ tertile & $25.80 \%$ & $33.33 \%$ & $1.17(0.36-3.74)$ & 0.796 \\
\hline $3^{\text {rd }}$ tertile & $41.10 \%$ & $33.33 \%$ & $14.17(5.08-39.51)$ & $<0.001$ \\
\hline
\end{tabular}

